\title{
Zum Beginn der Pollensaison 2003
}

$\mathrm{R}$ und 8 Millionen Menschen sind in Deutschland gegen Pollen sensibilisiert. Für diese Zeitgenossen hat in diesen Tagen wieder die Leidenszeit begonnen wenn der Nichtallergiker die Frühjahrssonnenstrahlen begrüßt, sucht sich der Patient unseres Fachgebietes seinen persönlichen Kompromiss zwischen tolerablen Symptomen, Therapieintensität und Präventionsstrategien.

Vielleicht sucht dieser Zeitgenosse aber auch einen kompetenten Kollegen auf, der ihn davon überzeugen kann, eine optimale - statt minimale - Therapie durchzuführen, mit

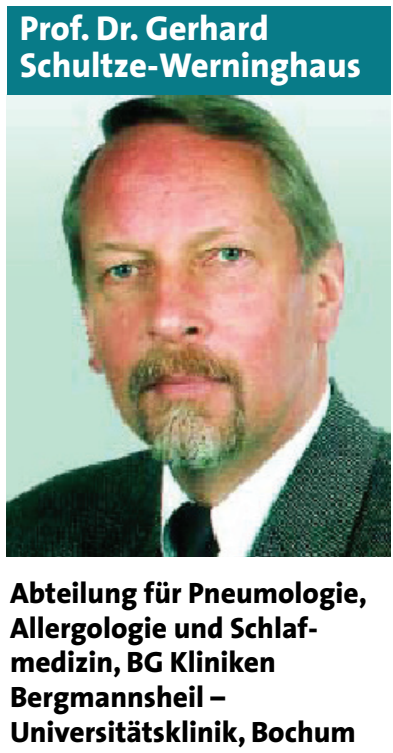

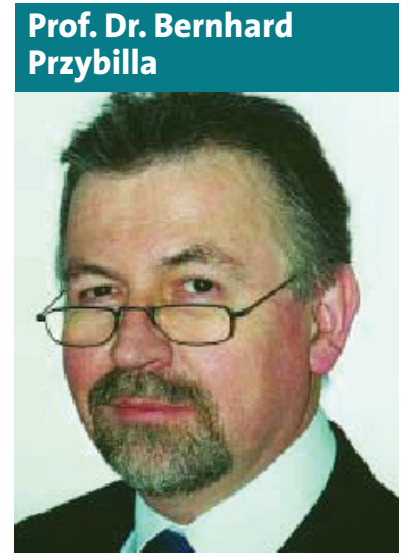

Klinik und Poliklinik für Dermatologie und Ludwig-MaximiliansUniversität, München Allergologie, Klinikum der

digkeit und Konzentrationsstörungen führen. Dieses Risiko war bei älteren Antihistaminika besonders hoch. Auf die aktuelle Situation sind derartige Befürchtungen aber nicht mehr übertragbar - im Gegenteil: eine adäquate Heuschnupfentherapie reduziert offenkundig die Unfallgefährdung, verglichen mit schlecht oder gar nicht behandelten Allergikern. Eine Behandlung des Heuschnupfens mit angemessenen Medikamenten (neuere, nicht-sedierende Antihistaminika, lokal wirksame Glukokortikoide) bessert nicht nur die Symptomatik, sondern auch der Aussicht auf eine bestmögliche Unterdrückung allergischer Symptome, eine Verhinderung zunehmender Asthmasymptome und damit eine bestmögliche Langzeitprognose. Diese Therapie würde vor allem auch eine subkutane spezifische und perenniale Immuntherapie über einen Zeitraum von drei Jahren einschließen. Inwieweit die staatlichen Bemühungen um eine Gesundheitsreform - wie die Vorschläge der Rürup-Kommission oder die

ptome an Nase und Augen auf, sondern es kann auch zu einer erheblichen Beeinträchtigung der Lebensqualität, zu Müdigkeit und Konzentrationsstörungen kommen. Von besonderer Bedeutung für die Leistungsfähigkeit und die Unfallrisiken im Straßenverkehr sowie am Arbeitsplatz ist, dass bei einer allergischen Rhinitis nicht nur Schlafstörungen infolge lästiger $\mathrm{Be}$ schwerden auftreten, sondern auch schlafbezogene Atmungsstörungen, die die Versorgung von chronisch Asthmakranken - beziehungsweise die verfügbaren Budgets der niedergelassenen Kollegen es tatsächlich erlauben werden, diese Evidenz-basierten Therapiekonzepte umzusetzen, wird sich zeigen. DGAI und ÄDA haben die betreffenden Gremien vielfach informiert - ob erfolgreich, wird sich zeigen. Skepsis ist angesagt.

Menschen mit allergischen Krankheiten der Atemwege können in ihrem Wohlbefinden erheblich beeinträchtigt sein. Dies gilt auch für den oft fälschlich als Bagatelle angesehenen „Heuschnupfen". Es treten nicht nur SymDisease-Management-Programme für

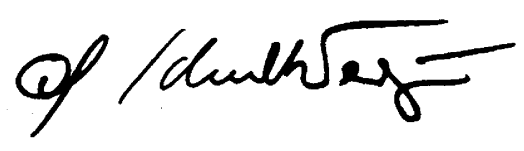

Prof. Dr. G. Schultze-Werninghaus die Lebens- und Schlafqualität, die Vigilanz wird durch neuere Antihistaminika im Vergleich zu unbehandelten Patienten mit Heuschnupfen sogar gesteigert. Daher ist es wichtig, dass auch relativ geringe Symptome allergischer Atemwegskrankheiten adäquat therapiert werden, um derartige Ausfallserscheinungen zu verhindern.

Die Deutsche Gesellschaft für Allergologie und klinische Immunologie hat in diesem Jahr, unterstützt durch $\mathrm{ADAC}$ und AvD, darauf hingewiesen, dass durch Unter- und Fehlbehandlung allergischer Atemwegserkrankungen ein erhöhtes Unfallrisiko im Straßenverkehr besteht und dass daher Allergiker zu einem verantwortungsbewussten Umgang mit ihrer Erkrankung verpflichtet sind. Ziel dieser Kampagne ist es, eine konsequente und bestmögliche Behandlung allergischer Atemwegserkrankungen zu erreichen.

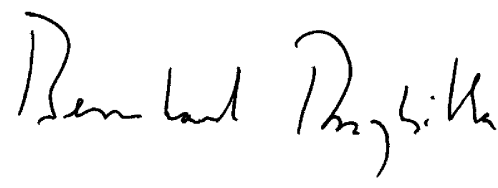

Prof. Dr. B. Przybilla 